\title{
De conscribendis epistolis (1522), ch. 49-50
}

Traduction : Philippe Collé et Christine Noille. Notes : Christine Noille

\section{Érasme}

Christine Noille (éd.)

Traducteur : Philippe Collé et Christine Noille

\section{(2) OpenEdition}

\section{Journals}

Édition électronique

URL : http://journals.openedition.org/rhetorique/540

DOI : $10.4000 /$ rhetorique. 540

ISSN : 2270-6909

\section{Éditeur}

UGA Éditions/Université Grenoble Alpes

Édition imprimée

ISBN : 978-2-37747-010-5

Référence électronique

Érasme, "De conscribendis epistolis (1522), ch. 49-50 », Exercices de rhétorique [En ligne], 9| 2017, mis en ligne le 20 juin 2017, consulté le 12 septembre 2020. URL : http://journals.openedition.org/ rhetorique/540; DOI : https://doi.org/10.4000/rhetorique.540

Ce document a été généré automatiquement le 12 septembre 2020.

\section{(c) (i) (2)(2)}

Les contenus de la revue Exercices de rhétorique sont mis à disposition selon les termes de la Licence Creative Commons Attribution - Pas d'Utilisation Commerciale - Partage dans les Mêmes Conditions 4.0 International. 


\title{
De conscribendis epistolis (1522), ch. $49-50$
}

Traduction : Philippe Collé et Christine Noille. Notes : Christine Noille

\author{
Érasme \\ Christine Noille (éd.) \\ Traduction : Philippe Collé et Christine Noille
}

\section{Édition}

Édition princeps : Érasme, De conscribendis epistolis, Bâle, Froben, 1522, ch. 49-50, p. 237-278 (sur Google Livres). Nous reprenons dans notre traduction le paragraphage de cette édition, dont nous indiquons entre crochets droits les pages : [p. 238], etc.

Texte établi dans : De conscribendis epistolis [1522], éd. J.-Cl. Margolin, dans Opera Omnia Desiderii Erasmi Roterodami, Ordinis Primis, Tomus Secundus, Amsterdam, North-Holland Publishing Company, 1971, p. 153-579, ch. 49-50, p. 432-466. Soit les colonnes 426-434 dans le tome I de l'édition des Opera, Leyde, P. van der Aa, 1703-1706 : nous indiquons, également entre crochets droits, cette référence internationale sous la forme « LB I 426 », etc.

Dans le chapitre 49 Érasme insère à titre d'exemples deux longues lettres de consolation : la Consolation à Canidius sur son exil (dans l'éd. de 1522, op. cit., p. 242-250) et la Consolation à Antoine Sucquet sur la mort de son fils (ibid., p. 250-266). Nous ne les avons pas traduites. Sont en revanche traduits tous les florilèges ainsi que les exemples brefs : ch. 49, Consolation à un proche avec objurgation, Consolation plaisante à un proche; ch. 50, Réponse à une lettre de consolation. Pour une traduction complète du De conscribendis epistolis, voir la traduction de Charles Fantazzi, On the 
Writing of letters, dans Collected Works of Erasmus, v. 25, éd. J. K. Sowards, University of Toronto Press, 1985, p. 148-189.

Nous avons choisi d'éditer en parallèle les chapitres sur la consolation de Vossius, de Scaliger et d'Érasme : nous nous sommes efforcés de traduire de la même façon d'un texte à l'autre les expressions latines qu'ils avaient en commun.

Pour une reprise des propositions qui sont formulées par Érasme dans le chapitre 49, voir G. J. Vossius, Rhetorices contractae, sive Partitionum Oratoriarum libri quinque ( $1^{\mathrm{e}}$ éd. Leyde, J. Maire, 1621), II, 24, édition et traduction dans le présent numéro, section ATELIER. Nous y renvoyons également pour la présentation générale et les commentaires en note.

\section{La lettre de consolation / CAP. XLIX. De consolatoria epistola}

1 Comme la vie des mortels est de toutes parts remplie de calamités et que rares sont ceux à qui il est permis de ne pas s'affliger de leur sort, nul devoir ne survient plus souvent que de soulager nos amis par des paroles de consolation. Et à vrai dire ce n'est pas un médiocre bienfait qu'une consolation à propos et amicale, par laquelle toutes les fois que dans les situations de détresse il n'est pas permis de remédier par des actions à la peine de ceux que nous aimons et voulons aider [quibus bene volumus ${ }^{1}$ ], du moins nous apaisons leur douleur par des mots. Il faut cependant le faire habilement, de peur que, semblables à des médecins inexpérimentés, nous n'aggravions une blessure encore à vif et toute fraîche au lieu de l'adoucir. En conséquence, il y aura trois façons de procéder pour faire une consolation ${ }^{2}$. Une première, simple et directe ${ }^{3}$ : nous faisons voir clairement à l'aide d'arguments qu'il n'y a pas de raison de souffrir, puisque rien ne peut attrister un homme sage [p. 238] sinon l'infamie et que nul ne peut le blesser que lui-même. Nous nous servirons de puissants remèdes de ce genre chaque fois que nous aurons affaire à un philosophe ou en général à un homme sensé. Il faut procéder autrement, par insinuation, quand l'âme de celui qui est affligé est trop faible ou la douleur trop récente ou trop aiguë pour souffrir d'être traitée directement: et de même quand l'âme de la personne que nous consolons est trop fière pour paraître avoir besoin qu'on la console. Car de même que certains individus sont dotés d'une âme si orgueilleuse que même lorsqu'ils sont gravement malades, ils ont honte d'appeler un médecin parce qu'ils considèrent que c'est lâche et humiliant pour un homme courageux d'être malade, il y a également des gens qui, en vertu d'une honte étreignant leur esprit généreux, étouffent et dissimulent tant et plus la grande douleur de leur âme. Avec ce genre de personnes il faudra s'avancer insensiblement, de cette manière : nous dirons que nous ne lui écrivons pas pour le consoler, car nous avons pu constater de multiples manières sa sagesse remarquable et son inébranlable grandeur d'âme, qui est plus forte que tous les orages de la fortune; et que même si nous admettons que son malheur est tel qu'il aurait facilement mis à terre n'importe qui d'autre, cependant nous ne doutons pas qu'un homme élevé depuis l'enfance dans les préceptes de la vraie philosophie, instruit par une longue expérience, et muni, contre ces épreuves, d'une force de caractère invincible supportera avec courage ce qui ne peut être évité, puisque c'est là la loi commune qui s'impose aux mortels. Nous dirons que nous voulons le 
féliciter pour sa fermeté, plutôt que remédier à sa douleur. Dans le premier cas, nous remédierons en transférant sur nous-mêmes les sentiments de la personne que nous voulons consoler, adaptant [attemperantes] notre discours [p.239] de sorte que nous paraissions vouloir plutôt céder à notre douleur qu'adoucir sa peine. C'est de cette manière que Cicéron, dans sa plaidoirie pour Milon, feint de transférer sur lui la crainte pour l'ôter de l'esprit des juges ${ }^{4}$. Car ceux que la douleur possède tout entiers ne doivent pas être traités différemment de ceux qui, du fait d'un manque de jugement dû à la maladie, pensent qu'ils ont des cornes, un nez proéminent par sa longueur, qu'ils sont morts ou qu'ils sont en terre cuite. Ils détestent ceux qui les contredisent et aiment ceux qui, par dissimulation, acquiescent à leurs chimères. Par conséquent, ceux qui s'efforcent de leur apporter des remèdes simulent parfois qu'ils sont eux-mêmes victimes du même mal. Puis une fois qu'ils ont gagné leur amitié [beneuolentia], ils les persuadent aisément du remède, et avançant peu à peu ${ }^{5}$, ils leur ôtent à la longue leurs chimères. C'est comme cela qu'il faudra s'y prendre avec ceux qui n'admettent pas encore la main du médecin. Nous dirons que nous sommes bien peu propres à consoler, nous qui ressentons autant de tourments que la personne que nous devons affermir. Puis nous outrerons aussi par une amplification les raisons de la douleur, ce que nous tirerons non seulement de la chose même, mais aussi du traitement indigne que subit celui qui souffre; et nous dirons, non sans quelque éloge, que nombre de gens de bien souffrent de l'injustice qui l'accable. Une fois que cela aura été fait, nous appliquerons le remède; à cet endroit nous rassemblerons avec soin tous les arguments qui sont susceptibles d'apaiser la douleur. Cela sera réalisé si nous atténuons avec vraisemblance le malheur même dans lequel il est tombé et [LB I 427] si nous minimisons, autant qu'il est possible, le bonheur d'où il est tombé. Nous atténuerons le malheur si nous montrons qu'il sera de courte durée. [p. 240] Car nous supportons habituellement avec plus de patience les infortunes que nous pensons devoir être bientôt finies, surtout s'il est montré que cette infortune sera suivie de quelque grande espérance. À ce point nous examinerons avec le plus grand soin toutes les conjectures par lesquelles nous rendrons cette espérance au plus haut point probable. Pour ce qui est des sources d'où tirer les conjectures, les préceptes des rhéteurs concernant l'état de cause conjectural les décrivent très clairement ${ }^{6}$; et beaucoup de lettres de Cicéron peuvent servir de modèles, en particulier la lettre à Cécina dans laquelle, il fait des conjectures dans le style plein d'esprit qui est le sien, en paraissant transmettre sa science de l'augure ${ }^{7}$. À tout cela, nous mêlerons quelque flatterie.

2 Mais si l'infortune semble du genre à devoir durer, nous userons d'autres façons de procéder pour l'atténuer ${ }^{8}$. Nous nous efforcerons alors de montrer soit que ce n'est pas du tout une infortune, soit qu'elle n'est pas aussi grave qu'il y paraît. On le fera en mentionnant précisément, avec quelque amplification, tous les avantages qui en résulteront. Car il n'y a pas de mal si grave qui puisse survenir dans la destinée humaine qu'on ne puisse en récolter un avantage. Si nous disons que tout ce qui atteint un homme sans que ce soit sa faute ne saurait être regardé comme un mal, comme l'enseigne Aristote 9 , selon lequel doux est le souvenir des maux passés quand ils sont associés à une réputation honorable, alors nous supporterons avec plus de légèreté les épreuves qui ne sont pas imputables à notre propre faute. C'est ici que les arguments des philosophes et particulièrement des stoïciens seront d'une très grande aide, si nous lui montrons qu'un tel malheur bénéficiera à sa réputation ou est un jalon posé dans la direction d'un très grand avantage ${ }^{10}$. [p. 241] En effet, la plus grande vertu et la plus grande félicité commencent le plus souvent avec les débuts les plus difficiles; et nous le 
prouverons par des exemples, si nous disons, à la façon des théologiens, que la tempête envoyée par la faveur divine est assurément fâcheuse pour le corps, mais salutaire pour l'âme. Rien ne soulage autant la douleur, selon Cicéron ${ }^{11}$, que de se rappeler notre commune condition qui est en quelque sorte la loi humaine ; c'est pourquoi nous ferons voir clairement que ce malheur, il le partage avec tout le genre humain, ou qu'il le partage assurément avec une foule d'hommes réputés et remarquables. À ce point, nous multiplierons les exemples d'hommes célèbres qui ont subi un chagrin [luctus] identique, ou du moins comparable, et l'ont supporté avec un courage exemplaire, pour leur plus grande gloire. Enfin nous en viendrons à l'exhortation : nous l'inviterons à renouer avec son ancienne constance, sa grandeur d'âme ${ }^{12}$, sa sagesse, sa culture. En conclusion nous offrirons toute notre assistance, notre zèle et notre soin pour lui, pour ses enfants et pour sa famille. Dans la consolation la plaisanterie sera absolument à proscrire, à moins qu'il ne s'agisse d'une légère infortune, et dans ce cas on pourra le dérider avec une plaisanterie. Si nous écrivons ainsi, pourvu que celui à qui nous écrivons soit du genre à être charmé par de telles choses, il faudra systématiquement plaisanter en caressant de sorte que nous mêlerons néanmoins de véritables consolations aux plaisanteries. En outre, nous prendrons garde de ne pas paraître donner des leçons [praecipere] plutôt qu'une consolation amicale, sauf si la personne à qui nous écrivons est un proche; de ne pas donner des ordres [imperemus ${ }^{13}$ ] comme le bien portant aux malades; de ne pas rappeler notre propre bonheur ou celui des autres; de ne pas imiter grossièrement le Tityre de Virgile : "Ô Mélibée, c'est un dieu qui nous a fait ce sort tranquille ${ }^{14}$.» [p. 242] Car de même que savoir le malheur partagé l'allège, le rappel de l'immunité dont jouit un autre exaspère la peine. Comme je pense que nous avons assez donné d'attention aux préceptes, nous ajouterons un exemple. Canidius $^{15}$, un homme de grand renom à Rome, chassé par la haine des factions hostiles, fut exilé dans de lointaines contrées, abandonnant derrière lui sa femme et ses jeunes enfants ; un ami lui adresse cette consolation ${ }^{16}$. [....

[p. 250] [LB I 430] Bien que la consolation soit surtout pratiquée pour les deuils et l'exil, il sera permis d'imaginer d'autres sujets concernant les divers événements qui sont une cause d'ennuis, tels que la maladie, une santé fragile ou précaire, la vieillesse, un mariage qui ne laisse rien augurer de bon et dont on se repent en vain, rentrer dans les ordres monastiques, revêtir la prêtrise ou tout autre genre de vie dont on finit par se dégoûter: les infortunes de sa lignée, la bassesse de sa condition, une pauvreté fâcheuse, la haine, la perte brutale d'un bien, des services rendus à un ingrat, des enfants qui déshonorent leurs parents par une vie dépravée, la peste, la guerre, et d'innombrables autres choses. J'ai cru bon de les mentionner pour les maîtres de rhétorique, comme autant de sources où puiser de nouveaux sujets d'exercices.

Nous ajouterons encore un second exemple ${ }^{17}$. Antoine Sucquet a perdu son fils, jeune homme plein de promesses. Un ami lui adresse cette consolation ${ }^{18}$. [...]

\section{Florilège $^{19}$}

[p. 266] [LB I 430] « Ainsi donc point de regret, point d'abattement. Ne souffrez pas que la multitude des affaires devienne un torrent qui vous accable; dressez-vous résolument pour leur faire tête, allez vous-même au-devant du flot ${ }^{20}$. » « Il n'y a pas de 
chagrin que le temps ne diminue et n'adoucisse à la longue ${ }^{21}$ ", et contre laquelle un cal se forme ${ }^{22}$. «Nous ne devons pas oublier que nous sommes hommes; que la loi de notre naissance est de vivre en butte à toutes les épreuves; que nous n'avons pas le droit de refuser la condition sous laquelle nous naissons et vivons; que nous ne devons pas surtout nous roidir contre ces coups de la fortune que nulle prévoyance humaine ne peut conjurer ; qu'en se rappelant ce qui est arrivé à autrui, on se convainc qu'il n'y a rien que d'ordinaire dans ce qui nous arrive à nous-mêmes ${ }^{23}$. " « Le sage doit s'affliger de la honte et non des revers, des fautes personnelles et non de l'injustice d'autrui ${ }^{24}$. " [LB I 431] Souviens-toi qu'il n'y a point « d'autre motif de consolation dans les grandes disgrâces que le témoignage d'une bonne conscience, et la persuasion que tous les maux sont légers pour celui qui n'a rien à se reprocher ${ }^{25}$. "

6 «Il ne faut pas qu'éprouvés comme nous le sommes par la pratique et le mouvement des grandes affaires, nous nous laissions aller au découragement ou à la faiblesse. La peine s'aigrit par les reproches qu'on peut se faire ${ }^{26}$.»

7 [p. 267] « Ayez courage et confiance. Votre adversaire est un homme bien léger. L'orage passera, et vous retrouverez et les égards qui vous sont dus, et la gloire qu'on vous dispute ${ }^{27} . »$

8 «Dans la douleur extrême que j'éprouve à votre sujet, je me console pourtant en pensant qu'infailliblement la sage conduite de vos amis et le temps qui affaiblit tout, jusqu'au venin de l'envie et de la haine, finiront par triompher de la perversité des hommes. C'est une consolation aussi pour moi que le souvenir de mes disgrâces dont je retrouve une image dans les vôtres ${ }^{28} »$.

9 Livre IV, la lettre : «Il ne se passe pas de jour qu'on ne me parle de votre trouble et de votre désespoir ${ }^{29} \ldots$ »; même lieu : «La mort de Tullie votre fille, dont on vient de me donner la nouvelle ${ }^{30} . . . » ;$ même lieu : «Comment vous donner un conseil ${ }^{31} . . . »$

10 "Je pourrais, il est vrai, chercher des consolations et vous indiquer des remèdes à vos souffrances. Mais s'il y a un homme capable de se faire une raison et de consoler les autres, n'est-ce pas vous? Je ne vous parlerai donc point de ce qu'on peut demander à la raison et à la science; vous le savez, et vous verrez ce qui sied aujourd'hui à un citoyen courageux et à un sage; vous verrez ce qu'exigent et la gravité de votre caractère et l'élévation de votre âme, et votre passé, et vos penchants, et tous ces dons par ou vous excelliez dès l'enfance ${ }^{32}$."

11 "Je vous conjure en finissant de ne pas perdre courage. Remettez-vous sans cesse en l'esprit et les exemples des grands hommes, et les principes que vous avez puisés dans l'étude et la méditation, rassemblez ainsi toutes vos forces : l'espérance vous sera plus douce, et l'avenir vous trouvera plus résigné. [p. 268] Mais je vous dis ce que vous savez mieux que moi, mieux que tout autre ${ }^{33}$.»

12 «Vous ne prétendez pas sans doute ni que la fortune fasse une exception pour vous, ni qu'elle vous sépare du sort commun ${ }^{34}$.»

13 Livre V, la lettre : «Personne au monde n'est moins en état que moi ${ }^{35} \ldots$.. ; même lieu : "Je veux vous consoler et j'aurais besoin moi-même ${ }^{36} \ldots$ "

14 «Mais nous avons joui de la prospérité avec modération; supportons avec fermeté, je ne dirai pas le changement, mais le renversement complet de notre fortune. Même quand on est heureux, on doit mépriser la mort, précisément parce que la mort est l'absence de tout sentiment. Dans l'excès de nos maux, instruisons-nous non seulement à la mépriser, mais encore à la désirer. Gardez-vous, croyez-moi, de renoncer à vos 
doux loisirs, et soyez-en bien convaincu : hors le vice, hors le mal dont vous êtes, dont vous serez toujours bien loin, il n'est rien sur la terre qui doive inspirer a l'homme de l'horreur ou de l'effroi ${ }^{37}$."

Livre VI, la lettre: "On se trouve partout si mal à l'aise au milieu de cette perturbation ${ }^{38} \ldots »$

16 « Si, au contraire, tout est bouleversé sans ressource, et si nous devons assister à ce jour funeste dont s'effrayait déjà $\mathrm{M}$. Antonius, lorsque sa sage perspicacité pressentait l'orage épouvantable qui devait éclater sur nos têtes, j'avoue que je n'ai à vous offrir qu'une consolation qui est misérable, surtout pour un citoyen et un homme tel que vous, mais qui cependant est la seule : c'est qu'on ne doit pas s'affliger pour soi d'un malheur qui frappe également sur tous. Je n'ajouterai rien de plus : si vous réfléchissez, comme je n'en doute pas, au sens profond de ce peu de mots ${ }^{39}$ ", etc.

17 « Vivre comme il faudrait vivre alors, c'est là le plus grand des maux. Aucun sage n'a dit que la mort fût un mal même pour l'homme heureux.

[p. 269] C'est ce que les murs même de la ville où vous êtes, vous diraient bien mieux et bien plus éloquemment que moi.

Je me borne donc, quoique la misère d'autrui soit une triste consolation, je me borne à vous affirmer de nouveau que vous n'êtes pas dans une position plus critique que qui que ce soit des nôtres, qu'il ait quitté le parti ou soit demeuré sous son drapeau. Les uns ont à combattre un ennemi, les autres à redouter un vainqueur; mais c'est là, je le répète, une triste consolation. En voici une meilleure : faites-en, comme moi, votre profit. Tant qu'on respire, si on n'a rien à se reprocher, on ne doit se tourmenter de rien. Quand on n'est plus, on est insensible à tout. Mais moi, vous parler ainsi ! me voici donc encore envoyant des hiboux à Athènes ${ }^{40}$. "

18 Livre VI, depuis la début de la lettre: «Je n'ai rien de nouveau à vous écrire ${ }^{41} \ldots$. » jusqu'à : «Si vous trouvez que j'augmente ici votre douleur ${ }^{42} \ldots$.. »

19 «À présent que vos vertus et votre considération vous rouvrent le chemin de vos foyers, il est d'un sage, [LB I 432] il est d'une âme forte d'oublier ce que vous perdez pour ne songer qu'à ce qui vous est rendu. Vous vivrez au milieu des vôtres, au milieu de nous ; vous avez acquis en estime plus que vous n'avez perdu en fortune. La fortune ! quelle jouissance peut-elle offrir, quand la république n'existe plus ${ }^{43}$ ?»

20 Livre VI, depuis le début de la lettre: «Réjouissez-vous, mon cher Balbus, réjouissezvous sans crainte ${ }^{44} \ldots$ » jusqu'à : «Je n'attends pas pour vous donner ces nouvelles ${ }^{45} . .$. »

21 Votre désastre me tourmente violemment : mes propres infortunes ne me touchent que modérément ${ }^{46}$.

22 «Courage donc! courage! vous voyez que de motifs pour en avoir! D’ailleurs, après ce que vous avez fait, voulu, tenté pour la république, c'est pour vous une obligation de compter sur un meilleur avenir, ou du moins de vous résigner à l'adversité [p. 270] en homme qui n'a failli à aucun devoir, à aucune prévision, et qui a sa fermeté et son courage à opposer aux coups du sort ${ }^{47}$. "

23 «Ainsi, courage! courage et fermeté ! vous avez conservé votre sang-froid pendant la tempête, vous pouvez vous réjouir en voyant le calme prêt à renaître ${ }^{48}$. "

24 "Je vous redirai, quant au reste, ce que je vous ai déjà dit : soyez sûr que vous n'avez dans ce grand débat rien à redouter qui ne vous soit commun avec la patrie. La situation est affreuse sans doute. Mais quand on a vécu comme nous et quand on est 
parvenu à l'âge où nous sommes, on doit s'armer d'une courageuse résignation contre les maux qu'on n'a pas à se reprocher ${ }^{49}$."

«Mais pourtant je trouve une grande consolation à sentir ma conscience nette, surtout quand j'ai deux points d'appui tels que ma passion pour les lettres et la gloire de mon nom. La première, je ne la perdrai qu'avec la vie ; la mort même ne me dépouillera pas de la seconde ${ }^{50}$.»

Livre IV, la lettre : «Votre lettre me charme ; j'aime surtout cette tendre amitié ${ }^{51} . .$. »

« Mais point d'alarme! Je vous connais, et je sais combien on est prompt à s'inquiéter quand on aime. L'affaire est, je crois, de celles où il y a plus de bruit que d'effet ${ }^{52}$.»

«Il n'y a qu'une chose à se dire, c'est qu'on est homme, et qu'on doit se résigner aux conditions de l'humanité53.»

Des Lettres à Brutus, toute la lettre, si élégante : « Je voudrais, à mon tour, vous apporter les consolations que j'ai reçues de vous dans une épreuve semblable ${ }^{54} \ldots$ »

\section{Parmi les lettres de Pline le Jeune ${ }^{55}$}

30 [p. 271] «Voilà les motifs qui m'obligent à pleurer auprès de vous sa mort comme un deuil prématuré, si du moins il est permis de la pleurer ou même d'appeler mort, cette disparition qui met un terme plutôt à la condition mortelle de ce grand homme qu'à sa vie. Car il vit et vivra toujours et même de plus en plus présent à la mémoire des hommes et mêlé à leurs discours, depuis qu'il a disparu à leurs yeux. J'aurais voulu vous parler de beaucoup d'autres choses, mais je ne puis détacher mon esprit de cette unique contemplation : c'est à Verginius que je pense, Verginius que je vois, Verginius que des images vaines désormais, vivaces cependant, me montrent : je l'entends, je lui parle, je le tiens dans mes bras; des hommes qui l'égalent en mérite, nous en avons et nous en aurons d'autres, mais aucun n'égalera sa gloire ${ }^{56}$.»

31 "Une blessure encore fraîche redoute la main du médecin, puis elle la supporte et même la réclame; ainsi une douleur récente repousse et fuit les consolations, puis les désire et, si elles sont apportées avec douceur, y trouve un apaisement ${ }^{57}$. »

32 «Je suis profondément affligé que vous ayez perdu un disciple de la plus haute espérance. Sachant avec quelle exactitude vous remplissiez tous vos devoirs, et quel attachement vous avez pour ceux que vous estimez, je ne m'étonne point que sa maladie et sa mort aient dérangé vos études ${ }^{58}$.

«Je vous donne en ce moment les mêmes consolations dont je me sers pour me fortifier et me soutenir moi-même ${ }^{59}$.» "Les belles-lettres sont pour moi une jouissance et une consolation. Il n'est rien de si agréable qui le soit plus qu'elles ; il n'est rien de triste qui ne devienne moins triste par elles ${ }^{60}$. " «C'est une consolation et à la fois une cause de tourment ${ }^{61}$.»

\section{Florilège de ma façon pour les lettres de consolation ${ }^{62}$}

[p. 272] « Si la pensée commune selon laquelle vous n'êtes plus l'homme que vous étiez signifie quelque chose, il n'y a pas de raison de vouloir vivre. Mais même si cette pensée ne peut vous déplaire totalement, considérez non seulement qui vous étiez tout récemment encore, mais qui vous étiez jadis; ne vous préoccupez pas tant d'où vous êtes tombé, mais aussi d'où vous vous êtes élevés. » 
35 «Que l'homme reste étranger à ce propos efféminé : “le temps que ma destinée m'enseigne à pleurer ma défaite ${ }^{63}$ ". Bien au contraire, la raison devrait vous rendre invincible face à tous les hasards de la vie, de sorte que vous chassiez la douleur de votre esprit, au lieu de vous y habituer. »

«Pour vous, la conscience du devoir et le jugement des gens de bien devraient compter davantage que les propos des envieux. » «Le poids du témoignage rendu par un petit nombre d'hommes remarquables et [LB I 433] jouissant d'une grande considération doit vous réconforter davantage que les fables des foules ${ }^{64}$.»

38 "Vous supporterez cela plus aisément si vous enjoignez à votre esprit de considérer ce que la postérité dira de vous plutôt que votre propre siècle, dont la malveillance ôte tout jugement. » « Il me semble que l'injustice que vous ont faite des rivaux n'a pas tant enlevé à votre fortune, qu'elle n'a ajouté à la gloire de votre nom. »

40 «Je n'ai pas pris sur moi de devoir vous consoler parce que j'aurais jugé moins magnifiquement de la grandeur de votre âme, mais je l'ai fait parce qu'il arrive que les hommes d'un grand courage ne peuvent vaincre les assauts de la fortune, de même que parfois les meilleurs marins ne peuvent vaincre la fureur de la tempête ${ }^{65}$. "

41 «Je n'ignore pas le terrible coup que vous avez subi avec la perte de votre femme. [p. 273] Vous avez perdu une épouse dont la vertu exemplaire eût été admirée même parmi les anciens ${ }^{66}$. Quel respect n'avait-elle pas pour son mari, elle qui était si digne d'être respectée ${ }^{67}$ ! Que de vertus éminentes, propres aux différents âges et dispersées dans toutes les femmes de bien, se réunissaient et s'associaient en elle ${ }^{68}$ ! Cependant ce doit être pour vous une grande consolation que d'avoir si longtemps possédé un tel trésor ${ }^{69}$, car elle a vécu avec vous quarante-quatre ans sans querelle ni offense ${ }^{70}$. "Mais c'est d'autant plus dur à supporter qu'elle a été si longtemps avec moi !", me direzvous. Car il est plus facile d'oublier les plaisirs que nous avons seulement eu le temps de goûter ${ }^{71}$. Prenez garde de ne pas être un ingrat, en ne pensant qu'à ce que vous avez perdu et en ne vous souvenant pas du temps qu'il il vous a été donné de jouir de sa présence. Assurément c'est à votre prudence de ne pas permettre à la raison d'abdiquer toute influence sur votre esprit, ce qu'il faut du reste attendre surtout de la nécessité, du temps et de l'épuisement de la douleur ${ }^{72}$.»

42 «La douleur nous est commune. Vous avez perdu vos petits-fils, moi-même mes arrière-petits-fils. Mais je forme l'espoir que d'autres enfants naîtront, qui changeront notre douleur en joie, puisque la joie est préservée dès lors qu'il est permis d'avoir cet espoir.»

43 «Je diffère grandement de ceux qui pensent qu'il faut laisser à lui-même un homme en proie à une grande douleur, jusqu'à ce que la blessure devienne moins vive avec le temps et admette les soins. Quant à moi, autant je suis d'avis que la première chose est d'avoir un esprit par avance fortifié, comme par un antidote, par les préceptes de la philosophie contre tous les coups de la fortune, autant je crois que la seconde est d'attaquer immédiatement la douleur, de sorte qu'elle ne puisse devenir résistante à tous les remèdes, à la façon d'une maladie chronique. »

44 [p. 274] « Vous dites dans votre lettre que la douleur que vous avez contractée depuis la mort de votre femme non seulement n'est pas apaisée par le temps mais croît de jour en jour. " 
"Je considère plutôt que, comme dans les maladies chroniques, il faut avoir recours à toute espèce de remède qui puisse apporter un changement. Il y a les bains, la campagne, les affaires, l'étude des belles-lettres, les voyages, les spectacles, la compétition avec des adversaires, l'exercice d'une magistrature publique. Parmi ces expédients ou d'autres semblables, vous devez tantôt en essayer un, tantôt un autre. Peut-être découvrirez-vous de quoi guérir la peine qui a envahi votre esprit, ou alors de quoi l'extraire, pour ainsi dire, clou après clou ${ }^{73}$.» [...]

\section{Exemple de ma façon ${ }^{74}$ : Consolation à un proche avec objurgation}

Vous, vous tourmenter ainsi, languir ainsi? Pourquoi enfin vous désespérer, vous consumer en larmes? Cela adoucira-t-il le mal, plutôt que de l'exacerber? Pourquoi vous lamenter si pitoyablement sur ce qui ne peut être changé ? Qu'en est-il, à l'heure qu'il est, de votre fermeté d'âme, qu'en est-il de votre culture, qui vous servaient d'ordinaire à soulager la souffrance des autres ? Vous avez su guérir les autres, mais à vous-même vous n'avez été d'aucune utilité. Vous devez maintenant être votre propre médecin. Pourquoi vous épuisez-vous vainement en larmes, et pourquoi épuisez-vous vos amis par vos plaintes [querelis]? Qu'est-ce que cette impuissance, qu'est-ce que cette mollesse ? Avez-vous oublié que vous êtes un homme ? Cela vous pèse-t-il tant ? Comme si ce n'était jamais arrivé qu'à vous seul, ou que c'est d'une telle ampleur, qu'il n'a pas pu arriver à d'autres quelque chose de bien pire. Qu'y a-t-il de plus indigne de votre courage et de votre savoir, [p. 275] que d'être tourmenté, brisé, abattu, à un point où même une femme ne saurait l'être. Je ne vois pas, en effet, quel profit peuvent vous apporter des larmes vaines et des plaintes [querimoniae]. Car, si vous estimez votre mal tel que vous ne puissiez plus redevenir bien portant, pourquoi redoublez-vous cet inconvénient par votre chagrin? S'il y a un espoir, pourquoi ne vous appuyez-vous pas sur lui, et ne faites-vous pas ce qu'il faut pour échapper à ces maux dès que possible ? Si vous désespérez, songez que dans une telle situation le courage diminue le mal de moitié. Mais si vous espérez, pourquoi vous minez-vous comme si la situation était désespérée ? On me dit que vous êtes dans de tels tourments que le chagrin en arrive à égarer votre esprit. [LB I 434] Mais, au nom de Dieu! Que voulez-vous? Pourquoi ne vous endurcissez-vous pas plutôt, et ne vous préservez-vous pas pour des jours meilleurs? Pourquoi augmentez-vous le chagrin de vos amis, comblez-vous de plaisir vos ennemis, et vous laissez-vous aller d'une manière si indigne?

Mais toi, avec plus d'audace, va

Où ta fortune te le permet ${ }^{75}$.

Les destins conduisent celui qui se soumet à leurs arrêts ; ils entraînent celui qui

résiste $^{76}$.

Quant à vous, tant que votre conscience, votre courage, votre vertu, votre culture, votre connaissance du passé, vous accompagneront, ne vous considérez pas comme un exilé $^{77}$, où que ce soit, sous quelque lieu que vous viviez, même si vous êtes relégué audelà du pays des Gètes ${ }^{78}$. Mais pourquoi donner des leçons à Minerve ${ }^{79}$ ? Ce n'est pas parce que je suis bavard, mais votre ami dévoué [sed beneuolentia], que mes lettres sont trop longues. Je vous souhaite de recouvrer votre santé. 


\section{Consolation plaisante à un proche ${ }^{80}$} lettre de consolation, on tirera conseil de la situation elle-même. Car nous le remercions d'abord vivement pour ses bons services; puis nous louons son talent, disant que rien n'aurait pu être allégué pour apaiser la douleur, qu'il ne l'ait exprimé avec la plus grande éloquence ; et enfin que son discours sage, cultivé, solennel et pardessus tout amical a ôté une bonne part de notre tristesse ou l'a entièrement chassée ${ }^{83}$. Ou alors, si sa consolation ne nous a fait aucun bien, nous louerons son intention, son zèle et son talent : et nous dirons qu'à l'égal d'un bon docteur plein d'expérience, il n'y a pas un remède qu'il n'ait appliqué, mais que le mal était trop grand pour l'art du médecin $^{84}$. [p. 277] Ou alors nous répondrons ainsi à un correspondant qui nous donne des ordres [imperioso $\left.{ }^{85}\right]$ : «Il est facile, quand on se porte bien, de donner de bons conseils aux malades. / Si tu étais à ma place, tu penserais autrement ${ }^{86}$. " l'adoucissement des peines de cœur que je n'aie lu chez vous. C'est en vain : la peine est la plus forte. J'ai fait plus, et sans doute personne avant moi n'en avait donné l'exemple : j'ai composé sur moi-même des lettres de consolation. J'attends qu'on en ait fini la copie pour vous les envoyer ${ }^{92}$.» 
«Mais trêve de consolations; faites-moi grâce de vos reproches. Car je me demande alors où est votre affection, où est votre sympathie, à vous que je crois cependant touché de mes disgrâces, à vous à qui je crois des consolations non moins nécessaires qu'à moi-même ${ }^{93}$ ?»

\section{Réponse de ma façon à une lettre de consolation ${ }^{94}$}

\section{NOTES}

1. Litt. " ceux dont nous voulons le bien ", formule verbale à laquelle correspond le substantif beneuolentia. Comme le précise J. Hellegouarc'h dans Le vocabulaire latin des relations et des partis politiques sous la République (Paris, Les Belles Lettres, 1963, p. 44 et 149-150), si amicitia et beneuolentia sont de quasi-synonymes, le second mot apporte comme nuance l'idée d'amitié active : d'où le sens intensif de « dévoué » que peut avoir l'adjectif beneuolus.

2. En marge (éd. 1522) : « Triplex ratio tractandae consolationis. »

3. L'adjectif aperta s'oppose, chez Érasme comme chez Vossius, à la manière « obliqua », « oblique, indirecte, détournée ».

4. Cicéron, Plaidoyer pour T. A. Milon, dans Euvres complètes de Cicéron, trad. P. C. B. Gueroult, sous la dir. de M. Nisard, Paris, Dubochet, t. III, 1843, voir en particulier l'entame: "Juges, il est honteux peut-être de trembler au moment où j'ouvre la bouche [...]».

5. L'adverbe paulatim est chez Quintilien un marqueur de l'insinuation.

6. Voir Cicéron, De inventione, 1.14 .19 ; Topica, 25.93 ; Quintilien, Institutio oratoria, 3.6.29 ; etc.

7. Cicéron, Familiares, 6, 6. Voir en particulier la transition centrale : «Maintenant donc qu'a la manière des augures et des astrologues, moi, qui suis augure aussi, je vous ai prouvé par des faits [en relatant des faits] ma science augurale et divinatoire, vous ne pouvez vous dispenser de croire à ma prédiction nouvelle. Je n'ai pas consulté le vol des oiseaux, je n'ai pas examiné si, suivant les règles sacramentelles de la discipline, leur chant vient de la gauche; je ne me suis arrêté ni aux miettes qui tombent, ni au son qu'elles rendent. J'ai consulté des signes qui, sans être absolument certains, permettent pourtant d'aller un peu moins à tâtons et trompent moins souvent que les autres. » Cécina avait écrit un traité de divination.

8. En marge (éd. 1522, qui va comme ici à l'alinéa) : «Alter modus extenuandi malum. »

9. Voir Vossius, Rhetorices contractae..., op. cit., §21. Aristote, Rhétorique, 1.11,1370a35, trad. P. Chiron, Paris, Flammarion, 2007, p. 215 : « Les choses dont le souvenir est plaisant, ce sont non seulement celles qui étaient plaisantes sur le moment, quand elles étaient présentes, mais pour certaines, des choses qui n'étaient pas plaisantes, si ce qui a suivi plus tard a été beau et bon, ce qui a fait dire : Il est plaisant, une fois sauvé, de se rappeler les dangers qu'on a courus [N.d.E. : Euripide, Andromède, fr. 131 Nauck] et: Car après coup, lorsqu'il se souvient, il prend plaisir même à ses souffrances, l'homme qui a beaucoup subi et beaucoup accompli [N.d.E.: d'après Homère, Odyssée, 
v. 400-401]. » À rapprocher de l'Éthique à Nicomaque, 1.11, $1100 \mathrm{~b} 9$ sq., trad. J. Tricot, Paris, Vrin, 1987, p. 73 sq.: «La cause véritablement déterminante du bonheur réside dans l'activité conforme à la vertu, l'activité en sens contraire étant la cause de l'état opposé. [...] [L'homme vertueux] supportera les coups du sort avec la plus grande dignité et un sens en tout point parfait de la mesure, si du moins il est véritablement homme de bien et d'une carrure sans reproche [N.d.E. : vers de Simonide]. » Argument récurrent du stoïcisme antique. Voir à titre d'exemple Sénèque, Lettre 74 à Lucilius (dans Les Stoïciens, trad. E. Bréhier, éd. sous la dir. de P.-M. Schul, Paris, Gallimard, "La Pléiade », 1962, p. 793) : "Quiconque s'est beaucoup livré au jeu de la fortune s'est préparé de très grands, d'inextricables sujets de trouble: la seule voie pour se mettre en sûreté consiste à mépriser les choses extérieures et à se contenter de ce qui est honnête. Quiconque pense qu'une chose est meilleure que la vertu, ou qu'un autre bien existe, présente le pli de sa toge aux hasards de la fortune [...]. »

10. Soit les deux lieux majeurs du délibératif : l'honorable (ou la gloire), ici fama, puis l'utile, ici commodum.

11. Voir par exemple Cicéron, Familiares, 5.16.2 (trad. M. Defresne, sous la dir. de M. Nisard, Paris, Dubochet, t. V, 1843) : «Nous ne devons pas oublier que nous sommes hommes; que la loi de notre naissance est de vivre en butte à toutes les épreuves; que nous n'avons pas le droit de refuser la condition sous laquelle nous naissons et vivons [...].» Ou encore, cité par Vossius (Rhetorices contractae..., op. cit., § 28), Cicéron, Familiares, op. cit., 5.16: « [...] C'est de vous rappeler que vous êtes homme, et homme de courage; que comme tel vous devez vous résigner aux chances communes de l'humanité, et supporter en sage ce qu'il n'était au pouvoir de personne de prévenir ou de détourner. » Pour d'autres exemples, voir infra, «Florilège ».

12. Litt. « de la grandeur» (magnitudinem). Au vu du contexte, cela ne peut signifier que animi magnitudinem, syntagme déjà employé supra par Érasme (éd. 1522, p. 238, «sa sagesse remarquable et son inébranlable grandeur d'âme ", « infractam animi magnitudinem »).

13. Voir infra au chap. 50 le même contexte et le même mot, contre le correspondant « qui nous donne des ordres» (imperioso). C'est prendre le ton du supérieur qui seul peut faire une objurgation.

14. Virgile, Les Bucoliques, 1.6. Traduction : Collection des Auteurs latins publiés sous la direction de M. Nisard, Lucrèce, Virgile, Valerius Flaccus, Paris, Didot, 1850.

15. En marge (éd. 1522, à Canidius) : «Argumentum siue thema epistolae. » Canidius est un nom fréquent à Rome. Lettre forgée par Érasme.

16. La lettre suit in extenso, sur neuf pages de l'éd. de 1522 (p. 242-250). En marge de l'édition, des manchettes repèrent les différentes parties, les sources des arguments et les figures de pensées.

17. Passage omis dans l'édition de Leyde de 1703-1706 ainsi que dans l'édition Froben des Opera omnia de 1540 ; la lettre est aux p. 435.20-440.33 de l'éd. Margolin.

18. Lettre forgée par Érasme. Première édition séparée en 1517, sous le titre Declamatio de morte, avec la Querela pacis. Dans le De conscribendis, la lettre suit in extenso, sur quinze pages de l'éd. de 1522 (p. 250-266 ; p. 441.12-455.27 de l'éd. Margolin). Comme pour le modèle précédent, en marge de l'édition des manchettes repèrent les différentes parties, les sources des arguments et les figures de pensées.

19. Ce premier florilège épistolaire (ou recueil de matériaux argumentatifs extraits d'œuvres modèles) est exclusivement cicéronien. Nous rajoutons les guillemets pour marquer le geste de la citation. Sauf mention contraire, nous reprenons les traductions de l'édition des CEuvres complètes de Cicéron, Paris, Dubochet, t. V, 1843 : Lettres à Quintus (trad. T. Savalète ; abrév. Ad Quint.) ; Lettres familières (trad. M. Defresne; abrév. Fam.) ; Lettres à Atticus (trad. M. Defresne et T. Savalète ; abrév. Ad Att.) ; Lettres à Brutus (trad. M. Defresne ; abrév. Ad Brut.).

20. Ad Quint., 1.1.4.

21. Fam., 4.5.6. 
22. Cette dernière séquence ( et cui non callum obducat») est peut-être inspirée des Tusculanes, 2.15.36: " et ipse labor quasi callum quoddam obducit dolori », « et par le travail même il se forme une espèce de calus, qui fait qu'on ne sent point la douleur ».

23. Fam., 5.16.2.

24. Fam., 5.17.5.

25. Fam., 6.4.2. Traduction de l'abbé Prévost (1 ${ }^{\text {ère }}$ éd. 1747, 5 vol. in-12).

26. Ad Quint., 1.1.2. Dans le texte de Cicéron, la dernière phrase est plus longue : « S'il est vrai que la peine s'aigrit par les reproches qu'on peut se faire, c'est moi qui ai le plus besoin d'être consolé.»

27. Fam., 1.5b.2.

28. Fam., 1.6.1.

29. Fam., 4.3 en entier (consolation à Servius Sulpicius sur les calamités publiques).

30. Fam., 4.5 en entier (consolation de Servius Sixticius à Cicéron sur la mort de sa fille Tullia).

31. Fam., 4.8 en entier (consolation à Marcellus sur les vicissitudes politiques).

32. Fam., 4.13.4.

33. Fam., 4.13.7.

34. Fam., 4.15.2.

35. Fam., 5.16 en entier (consolation à Titius sur la mort d'un de ses enfants).

36. Fam., 5.18 en entier (consolation à T. Fadius sur sa disgrâce).

37. Fam., 5.21.4.

38. Fam., 6.1 en entier (consolation à Aulus Torquatus sur son bannissement).

39. Fam., 6.2.2.

40. Fam., 6.3.3 ; le proverbe final est donné en grec par Cicéron et Érasme, qui le reprend dans ses Adages, I, II, 11, $\mathrm{n}^{\circ} 111$ (Ululas Athenas). Cet extrait de la lettre Fam. 6.3 est réparti par Érasme sur trois paragraphes.

41. Fam., 6.4.1. Traduction de l'abbé Prévost (1 ${ }^{\text {ère }}$ éd. 1747, 5 vol. in-12).

42. Fam., 6.4.2. Même traduction.

43. Fam., 6.11.2.

44. Fam., 6.12.1.

45. Fam.,6.12.3.

46. Cette phrase semble être d'Érasme (la note de l'éd. Margolin renvoie par erreur à Cicéron, Ad Att., 3.2).

47. Fam., 6.13.5.

48. Fam., 6.14.3.

49. Fam., 6.20.3.

50. Fam., 7.3.4.

51. Fam., 9.16 en entier (consolation plaisante à Papirius Paetus sur ses difficultés financières).

52. Ad Att., 2.24.1.

53. Ad Att., 15.1.1.

54. Ad Brut., 1.9 en entier (consolation sur la perte d'un proche ?)

55. Ce deuxième florilège épistolaire est dédié aux Lettres de Pline le Jeune (abrév. Pline). Nous rajoutons les guillemets pour marquer le geste de la citation. Nous reprenons les traductions de : 1) pour les livres I à V, Pline le Jeune, Lettres, trad. C. Sicard, Paris, Garnier, 1954 ; 2) pour les livres VI à X : Lettres de Pline le Jeune, trad. De Sacy, J. Pierrot et M. Gabaret-Dupaty, Paris, Garnier, 1920.

56. Pline, 2.1.10.

57. Pline, 5.16.11.

58. Pline, 7.30.1.

59. Pline, 8.10.3.

60. Pline, 8.19.1. 
61. Cette dernière séquence n'est pas de Pline, mais de Cicéron : Fam., 16.8.1.

62. Nous rajoutons les guillemets pour marquer le geste de reprise et d'auto-citation.

63. Virgile, Énéide, 4.434. Traduction : M.-A. Buxus et J. Poucet, L'Énéide Louvaniste éd., 1998, site itinera-electronica, URL : http ://agoraclass.fltr.ucl.ac.be/

64. Litt. «que les propos des foules stupides"; rappelons que nous donnons ici une traduction ancienne de Cicéron, en l'occurrence celle de T. Savalète en 1843.

65. Depuis « il arrive que les hommes d'un grand courage » jusqu'à « la fureur de la tempête » : reprise littérale de Cicéron, Ad Quint., 1.1.5.

66. Récriture d'une lettre de Pline le Jeune (avec transposition de l'ordre des phrases, passage de la $3^{\mathrm{e}}$ personne à la $2^{\mathrm{e}}$ et amplifications). Voir Pline, 8.5.1: «Amisit uxorem singularis exempli, etiam si olim fuisset », " Il a perdu sa femme dont la vertu eût été admirée même parmi les anciens ».

67. Voir ibid., 8.5.1: «Quam illa reuerentiam marito suo praestitit, cum ipsa summam mereretur!» "Quel respect n'avait-elle pas pour son mari, elle qui était si digne d'être respectée !»

68. Voir ibid., 8.5.1.: " quot quantasque uirtutes, ex diuersis aetatibus sumptas, collegit et miscuit!» "Que de vertus éminentes, propres aux différents âges, se réunissaient et s'associaient en elle! »

69. Voir ibid., 8.5.2. : "Habet quidem Macrinus grande solacium, quod tantum bonum tam diu tenuit ", «Sans doute c'est une grande consolation pour Macrinus, d'avoir si longtemps possédé un tel trésor."

70. Voir ibid. : «Vixit cum hac triginta nouem annis sine iurgio sine offensa. » «Leur union a duré trente-neuf ans, sans trouble et sans nuage » (litt., " sans querelle ni offense »).

71. Érasme reformule totalement une sentence de Pline (ibid., 8.5.2) : « Nam fruendis uoluptatibus crescit carendi dolor. " « Car, plus la possession a de charmes, plus la perte coûte de regrets. »

72. Voir ibid., 8.5.3 : «[...] quam nihil aeque ac necessitas ipsa et dies longa et satietas doloris inducit ", «[...] ce qu'il faut attendre surtout de la nécessité, du temps et de l'épuisement de la douleur »

73. «Clou après clou » : voir Cicéron, Tusculanes, 4.35 .75 ; Érasme, Adages, I, II, 4, n 104 (Clavum clavo pellere).

74. Lettre forgée par Érasme.

75. Virgile, Énéide, 6.95-96. Nous traduisons.

76. Sénèque, Lettres à Lucilius, 107, 11, trad. M. Charpentier et M. Lemaistre, Paris, Garnier, 1860.

77. Voir Sénèque, Consolation à ma mère Helvie, 8.1.

78. Référence à l'exil d'Ovide à Tomis en Scythie mineure, peuplée de Gètes.

79. C'est-à-dire à plus savant que soi. Voir Érasme, Adages I, I, 40, nº 40 (Sus Minervam).

80. Exemple également forgé par Érasme.

81. Proverbe cité dans Cicéron, Des vrais biens et des vrais maux, 5.1.3 ; Érasme, Adages, I, II, 52, $\mathrm{n}^{\circ} 152$ (Vivorum oportet meminisse).

82. En grec dans le texte d'Érasme. Voir Érasme, Adages, I, IX, 10, nº 810 (Flere ad novercae tumulum).

83. Pour une illustration de cette réponse, voir Cicéron, Fam., 4.6 (lettre d'ailleurs référencée dans le florilège ci-après).

84. Pour une illustration de cette réponse en dehors du corpus cicéronien, voir Ovide, Pontiques, 1.3 (réponse à la consolation de Rufin sur son exil).

85. Voir supra le même contexte et le même mot : «ne pas donner des ordres » (imperemus). Le correspondant prend le ton du supérieur qui seul peut faire une objurgation. Infra, c'est sans doute aussi le contexte de la première phrase de la «Réponse de ma façon à une lettre de consolation » : « Vos discours m'ordonnent » (iubet).

86. Térence, Andrienne, 2.1.9. Traduction : Henri Clouard, Paris, Classiques Garnier, s.d.

87. Nous rajoutons les guillemets pour marquer le geste de la citation.

88. Fam., 4.6 en entier (réponse à la consolation de Servius Sulpicius sur la mort de sa fille).

89. Fam., 5.13 en entier (réponse à la consolation de Lucceius).

90. Ad Att., 3.3. Il s'agit d'une lettre brève, citée en entier par Érasme. 
91. Ad. Att., 11.2.3.

92. Ad Att., 12.14.3. Érasme omet la proposition subordonnée finale. Litt.: «quem librum ad te mittam, si descripserint librarii », « dont je vous envoie le livre, dès qu'on en aura fini la copie ».

93. Ad Att., 3.11.2.

94. Exemple forgé par Érasme.

95. Le contexte est sans doute le même que supra pour « donner des ordres " (avec l'emploi du verbe imperare). La réponse à la lettre de consolation récuse le ton de commandement de celle-ci.

\section{AUTEURS}

\section{ÉRASME}

Desiderius Erasmus Roterodamus, $1467 ?-1536$ 